\title{
THE COFIBRE OF THE TRANSFER MAP
}

\author{
LARRY W. CUSICK
}

\begin{abstract}
Suppose a finite group $G$ acts freely on a finite complex $X$ with orbit space $B$. The cofibre $\mathscr{C}$ of the transfer map, is defined by the cofibre sequence $\Sigma^{0} B_{+} \stackrel{\text { tr }}{\rightarrow} \Sigma^{0} X_{+} \rightarrow \mathscr{C}$. We show that there is a spectral sequence $H_{G}^{p}\left(X ; \tilde{M} \otimes h^{q}\right) \Rightarrow$ $h^{p+q}(\mathscr{C})$ for any cohomology theory $h^{*}$, where $\tilde{M}$ is the reduced regular $\mathbf{Z}$-representation for $G$. As a special case we prove that $H^{*}\left(\mathscr{C} ; \mathbf{Z}_{2}\right)$ is a free $H^{*}\left(B: \mathbf{Z}_{2}\right)$-module on a zero-dimensional class for any two-fold cover
\end{abstract}

0. Introduction. If a finite group $G$ acts freely on a topological space $X$ with orbit space $B$ then the natural projection map $X \rightarrow B$ is a finite cover. Under reasonable assumptions there is an associated stable map tr: $\Sigma^{0} B_{+} \rightarrow \Sigma^{0} X_{+}$called the transfer that has proved useful in solving problems in homotopy theory and group actions. It is the purpose of this paper to provide means for computing the cohomology of the cofibre of tr. Specifically, if $X$ is finite, $\mathscr{C}$ is the spectrum defined by the mapping cone

$$
\Sigma^{0} B_{+} \stackrel{\operatorname{tr}}{\rightarrow} \Sigma^{0} X_{+} \rightarrow \mathscr{C}
$$

and $h^{*}$ is a generalized cohomology theory, then we prove that there is a convergent spectral sequence

$$
E_{2}^{p, q} \cong H_{G}^{p}\left(X ; \tilde{M} \otimes h^{q}\right) \Rightarrow h^{p+q}(\mathscr{C}),
$$

where the cohomology groups appearing on the left are equivariant cohomology with coefficients in the $G$-module $\tilde{M} \otimes h^{q}(\tilde{M}=$ right reduced regular representation for $G)$. In particular, when $h^{*}$ is ordinary cohomology we obtain an isomorphism

$$
H^{*}(\mathscr{C}) \cong H_{G}^{*}(X ; \tilde{M})
$$

In $\S 1$ we outline the construction of the transfer. That section is not meant to be a detailed discussion on the transfer, rather its purpose is to establish the notation used in the existence proof of the spectral sequence. In $\$ 2$ we prove the main theorem. The spectral sequence turns out to be a reindexed Serre spectral sequence for a certain relative fibration that arises from the construction of tr. The cohomology $h^{*}(\mathscr{C})$ turns out to have the structure of a $h^{*}(B)$-module. As a special case, if $X \rightarrow B$ is a two-fold cover, we show that $\mathscr{C}$ is the Thom space $T\left(-\xi_{L}\right)$, where $-\xi_{L}$ is the complementary bundle of $\xi_{L}=L \times{ }_{\mathbf{z}_{2}} X$ and $L$ is the nontrivial one-dimensional

Received by the editors January 13, 1984 and, in revised form, May 3, 1984. Presented August 16, 1984 at the AMS meeting, University of Oregon, Eugene, Oregon.

1980 Mathematics Subject Classification. Primary 55R20, 57S17.

1985 American Mathematical Society $0002-9939 / 85 \$ 1.00+\$ .25$ per page 
orthogonal representation of $\mathbf{Z}_{2}$. In particular, $H^{*}\left(\mathscr{C} ; \mathbf{Z}_{2}\right)$ is a free $H^{*}\left(B ; \mathbf{Z}_{2}\right)$-module on a zero-dimensional class.

The author would like to thank the referee for several helpful comments and suggestions and, in particular, for pointing out Corollary 2.11 , which replaces a somewhat weaker theorem in the original version of this paper.

1. The transfer map. We assume throughout that $G$ is a finite group of order $n$ acting freely and cellularly on a finite $\mathrm{CW}$-complex $X$ with orbit space $B$. The transfer map associated to the finite cover $p: X \rightarrow B$ is a stable map $\operatorname{tr}: \Sigma^{0} B_{+} \rightarrow$ $\Sigma^{0} X_{+}$, where $\Sigma^{0} X_{+}$is the suspension spectrum whose $n$th term is the space $S^{n} \wedge X_{+}$ and $X_{+}$is $X$ with a disjoint basepoint added. We shall outline a construction of $\operatorname{tr}$ (for fuller detail see [A or BG]).

Let $D^{l}$ denote the $l$-disc and $\stackrel{D}{D}^{\prime}$ its interior. The first step in the construction is to produce an embedding $e: D^{l} \times X \hookrightarrow \stackrel{\circ}{D}^{\prime} \times B$ which makes the following diagram commute:

$$
\begin{array}{ccc}
D^{l} \times X & \stackrel{e}{\hookrightarrow} & D^{l} \times B \\
p \pi_{2} \searrow & & \swarrow \pi_{2} \\
& B &
\end{array}
$$

where $\pi_{2}$ is the projection onto the second factor. This may be done as follows: For a right orthogonal representation $W$ of $G$, with dimension $m$, let $\xi_{w}$ denote the $m$-plane bundle $\bar{p}: W \times{ }_{G} X \rightarrow B$, where $\bar{p}(v, x)=p(x)$. The bundle $\xi_{w}$ represents a class $\alpha$ in $\widetilde{K O}(B)$ that has finite order.

This may be seen as follows: Let $B_{q} G$ denote the $q$ th filtration of $B G$ arising from the Milnor construction of a classifying space [M]. According to [At], $\widetilde{K O}(B G) \cong$ $\lim \widetilde{K O}\left(B_{q} G\right)$, and the image of the natural map $\widetilde{K O}(B G) \rightarrow \widetilde{K O}\left(B_{q} G\right)$ is finite. Now, $\alpha$ is in the image of $f^{*}: \widetilde{K O}(B G) \rightarrow \widetilde{K O}(B)$, where $f: B \rightarrow B G$ is the classifying map of the cover $p: X \rightarrow B$. Since $B$ is finite, $f$ factors through a map $\vec{f}$ : $B \rightarrow B_{q} G$; consequently, we have the commutative diagram

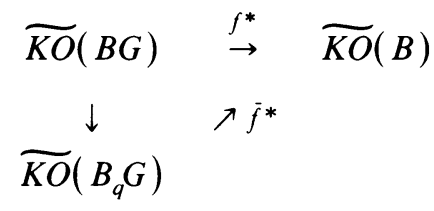

and $\alpha$ has finite order. This means there is a $k$ such that the direct sum $\oplus^{k} \xi_{w} \cong \xi_{\oplus^{k} w}$ is stably trivial over $B$. Let $R^{t}$ be a trivial representation such that $\xi_{V}$ is a trivial vector bundle, where $V=\oplus^{k} W \oplus R^{t}$. Thus, we may choose a trivialization

$$
\begin{array}{ccc}
D V \times{ }_{G} X & \underset{\phi}{\cong} & D^{\prime} \times B \\
\bar{p} \searrow & & \swarrow \pi_{2} \\
& B &
\end{array}
$$

where $l=\operatorname{dim} V$ and $D V$ is the unit disc in $V$. 
We now choose $W$ (hence $V$ ) to be faithful and orientation preserving (the latter assumption will be needed in §2). This allows us to equivariantly embed $j: D^{l} \times G$ $\hookrightarrow \stackrel{D}{D} V$. The embedding $e$ of (1.1) is given by the composition

$$
\begin{aligned}
D^{\prime} \times X \underset{\psi}{ } \underset{\underset{\psi}{\cong}}{\stackrel{\cong}{\rightarrow}}(D \times G) \times{ }_{G} X \\
\stackrel{j{ }_{G} X}{\rightarrow} D V \times{ }_{G} X \underset{\phi}{\stackrel{\cong}{\rightarrow}} D^{\prime} \times B,
\end{aligned}
$$

where $\psi(v, x)=(v, 1, x)(1=$ unit in $G)$. The commutative diagram (1.1) is easy to check.

The transfer may now be constructed as follows: Let $E=D^{\prime} \times B \backslash e\left(\stackrel{\circ}{D}^{\prime} \times X\right)$ and $E_{0}=\partial D^{l} \times B$. By collapsing $E / E_{0}$ to a point, we obtain the following map:

$$
\begin{array}{ccc}
D^{\prime} \times B / \partial D^{\prime} \times B & \stackrel{\Sigma^{\prime} \mathrm{tr}}{\rightarrow} & D^{\prime} \times X / \partial D^{l} \times X \\
S^{\prime} \wedge B_{+} & & S^{\prime} \wedge X_{+}
\end{array}
$$

In other words, the stable transfer is given by a cofibre sequence

$$
\Sigma^{-1} E / E_{0} \rightarrow \Sigma^{0} B_{+} \stackrel{\text { tr }}{\rightarrow} \Sigma^{0} X_{+} .
$$

For the uniqueness and naturality properties of tr one may consult, for example, [A].

(1.4) Definition. The cofibre $\mathscr{C}$ of $\mathrm{tr}$ is defined by the mapping cone

$$
\Sigma^{0} B_{+} \stackrel{\text { tr }}{\rightarrow} \Sigma^{0} X_{+} \rightarrow \mathscr{C} \text {. }
$$

(1.5) REMARK. By (1.3) we have an equivalence of spectra $\mathscr{C} \simeq \Sigma^{1-l} E / E_{0}$.

2. The cofibre of the transfer map. We now address the problem of computing $h^{*}(\mathscr{C})$ for any generalized cohomology theory $h^{*}$. From (1.5) we have

$$
h^{i}(\mathscr{C}) \cong h^{i+1-l}\left(E, E_{0}\right) \text {. }
$$

Let $F=D V \backslash j\left(\stackrel{D}{ }^{\prime} \times G\right)$ and $F_{0}=\partial D V$, where we are using the notation from $\S 1$. Note that the pair $\left(F, F_{0}\right)$ comes equipped with a right $G$-action.

For the notion of relative $h^{*}$-fibration see [D].

(2.2) Proposition. The projection map onto the second factor $\pi_{2}: E \rightarrow B$ (resp. $\pi_{2}$ : $\left.E_{0} \rightarrow B\right)$ is the projection map for a relative $h^{*}$-fibration $\left(E, E_{0}, \pi_{2}, B\right)$ with relative fibre $\left(F, F_{0}\right)$.

Proof. By definition of $\left(E, E_{0}\right), \phi$ induces a homeomorphism $\left(F \times_{G} X, F_{0}\right.$ $\left.\times_{G} X\right) \cong\left(E, E_{0}\right)$ over $B$. The proposition follows.

Thus, to compute $h^{*}\left(E, E_{0}\right)$ we may employ the relative Serre spectral sequence [D]

$$
E_{2}^{p, q} \cong H^{p}\left(B ; h^{q}\left(F, F_{0}\right)\right) \Rightarrow h^{p+q}\left(E, E_{0}\right) .
$$

The cohomology groups in the $E_{2}$-term are cohomology with a local coefficient system $h^{*}\left(F, F_{0}\right)$. 
Let $\tilde{G}$ be the fundamental group of $B$. If $X$ is connected there is an epimorphism $\varepsilon$ : $\tilde{G} \rightarrow G$, and so we may give $h^{*}\left(F, F_{0}\right)$ the structure of a $\tilde{G}$-module via $\varepsilon$. We call this new module $\varepsilon^{*} h^{*}\left(F, F_{0}\right)$. By a theorem due to $S$. Eilenberg (see [W]) we may identify

$$
H^{*}\left(B ; h^{*}\left(F, F_{0}\right)\right)=H_{\tilde{G}}^{*}\left(\tilde{B} ; \varepsilon^{*} h^{*}\left(F, F_{0}\right)\right),
$$

where the groups on the right represent equivariant cohomology with coefficients in the $\tilde{G}$-module $\varepsilon^{*} h^{*}\left(F, F_{0}\right)$.

(2.3) Lemma. If $M$ is a G-module with the induced $\tilde{G}$-module denoted by $\varepsilon^{*} M$ and $X$ is connected, then there is an isomorphism of equivariant cohomology groups $H_{\tilde{G}}^{*}\left(\tilde{B} ; \varepsilon^{*} M\right) \cong H_{G}^{*}(X ; M)$.

Proof. This is due to a natural isomorphism at the CW-cochain level. Let $\pi$ be the kernel of $\varepsilon\left(\pi \cong \pi_{1} X\right)$; then $\operatorname{Hom}_{Z[G]}\left(C_{*} X, M\right) \cong \operatorname{Hom}_{Z[G]}\left(Z \otimes_{Z[\pi]} C_{*} \tilde{B}, M\right) \cong$ $\operatorname{Hom}_{Z[\tilde{G}]}\left(C_{*} \tilde{B}, \varepsilon^{*} M\right)$.

By the above lemma we may identify

$$
H_{\tilde{G}}^{*}\left(\tilde{B} ; \varepsilon^{*} h^{*}\left(F, F_{0}\right)\right) \cong H_{G}^{*}\left(X ; h^{*}\left(F, F_{0}\right)\right) .
$$

It remains to describe the $G$-module structure of $h^{*}\left(F, F_{0}\right)$.

(2.4) Definition. Let $M$ (resp. $\tilde{M}$ ) denote the regular (resp., reduced regular) $Z$-representation of $G$.

(2.5) Lemma. Let $i: F_{0} \rightarrow F$ be the natural inclusion; then there is a commutative diagram of G-modules in which the vertical maps are isomorphisms, $\varepsilon$ is the augmentation map and $\tilde{h}^{*}\left(S^{l-1}\right)$ is a trivial G-module:

$$
\begin{array}{ccc}
\tilde{h}^{*}(F) & \stackrel{i^{*}}{\rightarrow} & \tilde{h}^{*}\left(F_{0}\right) \\
\cong \uparrow & & \uparrow \cong \\
M \otimes \tilde{h}^{*}\left(S^{l-1}\right) & \stackrel{\varepsilon \otimes 1}{\rightarrow} & \tilde{h}^{*}\left(S^{l-1}\right) .
\end{array}
$$

Proof. We first define the maps. Since $F_{0} \cong S^{l-1}$ and the representation $V$, which was used in the definition of $F_{0}$, is orientation preserving, $\tilde{h}^{*}\left(F_{0}\right)$ is a trivial $G$-module. Consequently, we may take the right-hand isomorphism to be the identity.

For each $g \in G$ let $F_{g}=D V \backslash j\left(\stackrel{D}{ }^{\prime} \times\{g\}\right)$, and $i_{g}: F \hookrightarrow F_{g}$ will be the natural inclusion. The natural inclusion $F_{0} \rightarrow F_{g}$ is a homotopy equivalence. Choose a homotopy inverse $\mu_{g}: F_{g} \stackrel{\sim}{\rightarrow} F_{0}$. The direct sum $\oplus_{g \in G} \tilde{h}^{*}\left(F_{0}\right)$ may be given the structure of a $G$-module and it is easy to check that the map

$$
\mu=\bigoplus_{g \in G}\left(\mu_{g} \circ i_{g}\right)^{*}: \bigoplus_{g \in G} \tilde{h}^{*}\left(F_{0}\right) \rightarrow \tilde{h}^{*}(F)
$$

is an isomorphism of $G$-modules. There is an obvious isomorphism $\alpha: M \otimes$ $\tilde{h}^{*}\left(F_{0}\right) \stackrel{\triangleq}{\rightrightarrows} \oplus_{g \in G} \tilde{h}^{*}\left(F_{0}\right)$ of $G$-modules. The left-hand isomorphism is the composition $\mu \circ \alpha$. The diagram may now be easily checked to be commutative. 
Since the inclusion $i: F_{0} \hookrightarrow F$ induces a split epimorphism on cohomology, we have

(2.6) LEMMA. The sequence

$$
0 \rightarrow h^{*}\left(F, F_{0}\right) \rightarrow \tilde{h}^{*}(F) \rightarrow \tilde{h}^{*}\left(F_{0}\right) \rightarrow 0
$$

is exact.

(2.7) Proposition. $h^{*}\left(F, F_{0}\right) \cong \tilde{M} \otimes \tilde{h}^{*}\left(S^{l-1}\right)$ as G-modules.

Proof. This follows from the following commutative diagram with exact rows and the previous two lemmas:

$$
\begin{aligned}
& \begin{array}{cccccc}
0 & \rightarrow & h^{*}\left(F, F_{0}\right) \\
\uparrow & \rightarrow & \tilde{h}^{*}(F) & \rightarrow & \tilde{h}^{*}\left(F_{0}\right) \\
& & \uparrow \cong & & \rightarrow \cong
\end{array} \\
& 0 \quad \rightarrow \quad \tilde{M} \otimes \tilde{h}^{*}\left(S^{l-1}\right) \quad \rightarrow \quad M \otimes h^{*}\left(S^{l-1}\right) \stackrel{\varepsilon \otimes 1}{\rightarrow} \quad \tilde{h}^{*}\left(S^{l-1}\right) \quad \rightarrow \quad 0
\end{aligned}
$$

It follows that we have the spectral sequence

$$
H_{G}^{p}\left(X ; \tilde{M} \otimes \tilde{h}^{q}\left(S^{l-1}\right)\right) \Rightarrow h^{p+q}\left(E, E_{0}\right) .
$$

Recalling that $h^{i}(\mathscr{C}) \cong h^{i+l-l}\left(E, E_{0}\right)$, we may reindex the above spectral sequence; letting $h^{q}=\tilde{h}^{q}\left(S^{0}\right)$ we have

(2.8) THEOREM. For connected $X$ there is a spectral sequence

$$
E_{2}^{p, q} \cong H_{G}^{p}\left(X ; \tilde{M} \otimes h^{q}\right) \Rightarrow h^{p+q}(\mathscr{C}) .
$$

(2.9) Corollary. $H^{*}(\mathscr{C} ; K) \cong H_{G}^{*}(X ; \tilde{M} \otimes K)$ for any commutative ring $K$.

REMARK. From the short exact sequence $0 \rightarrow \tilde{M} \rightarrow M \stackrel{\varepsilon}{\rightarrow} K \rightarrow 0$ we obtain a short exact sequence of cochain complexes

$$
\begin{aligned}
0 & \rightarrow \operatorname{Hom}_{K[G]}\left(C_{*}(X ; K), \tilde{M}\right) \rightarrow \operatorname{Hom}_{K[G]}\left(C_{*}(X ; K), M\right) \\
& \stackrel{\varepsilon_{*}}{\rightarrow} \operatorname{Hom}_{K[G]}\left(C_{*}(X ; K), K\right) \rightarrow 0 .
\end{aligned}
$$

We may identify $\operatorname{Hom}_{K[G]}\left(C_{*}(X ; K), M\right) \cong \operatorname{Hom}_{K}\left(C_{*}(X ; K), K\right)$ and $\operatorname{Hom}_{K[G]}\left(C_{*}(X ; K), K\right) \cong \operatorname{Hom}_{K}\left(C_{*}(B ; K), K\right)$, in which case $\varepsilon_{*}$ coincides with the transfer map tr. It follows that the long exact sequence arising from the above short exact sequence coincides with the sequence of the cofibration $\Sigma^{0} B_{+} \rightarrow \Sigma^{0} X_{+} \rightarrow$ $\mathscr{C}$.

We would like to remark that as a consequence of the definition of $\left(E, E_{0}\right)$ the groups $h^{*}\left(E, E_{0}\right)$ may be given the structure of an $h^{*}(B)$-module via the projection $\pi_{2}$. Thus, $h^{*}(\mathscr{C})$ may be regarded as an $h^{*}(B)$-module.

Consider the special case of a two-fold cover $p: X \rightarrow B$. We will show that in this case the cofibre of the transfer map is actually the Thom space of a certain bundle over $B$. Using the notation from $\S 1\left(G=\mathbf{Z}_{2}\right)$, we construct our equivariant embedding $j: D^{\prime} \times \mathbf{Z}_{2} \hookrightarrow D^{\prime} V$ so that it satisfies the properties

(1) $j(0, e)=-j(0, \tau)$, and

(2) lettting $L=$ one-dimensional subspace of $V$ that is the span of $j(0, e)(L \cong \tilde{M})$ and writing $V=L \oplus \tilde{V}$, we require that $j\left(D^{\prime} \times \mathbf{Z}_{2}\right) \cap \tilde{V}=\varnothing$. Now, $V \backslash \tilde{V}$ has two 
path components and $j\left(\stackrel{\circ}{D}^{\prime} \times\{e\}\right)$ and $j\left(\stackrel{D}{D}^{l} \times\{\tau\}\right)$ are in separate components. Consequently, the map $D \tilde{V} / S \tilde{V} \rightarrow F / F_{0}$, induced by the inclusion $D \tilde{V} \hookrightarrow F$, is a homotopy equivalence, and they are both equivalent to $S^{l-1}$.

Write $-\xi_{L}$ for the complementary bundle to $\xi_{L}=L \times \mathbf{z}_{2} X$, so $\xi_{L} \oplus-\xi_{L} \cong R^{\prime} \times B$. The Thom space $T\left(-\xi_{L}\right)$ is defined as

$$
D\left(-\xi_{L}\right) / S\left(-\xi_{L}\right) \cong D \tilde{V} \times \mathbf{z}_{2} X / S \tilde{V} \times_{\mathbf{z}_{2}} X .
$$

We define $i: T\left(-\xi_{L}\right) \rightarrow E / E_{0}$ by the commutative diagram

$$
\begin{array}{ccc}
T\left(-\xi_{L}\right) & \stackrel{i}{\rightarrow} & E / E_{0} \\
\| l & & \| l \\
D \tilde{V} \times \mathbf{z}_{2} X / S \tilde{V} \times \mathbf{z}_{2} X & \rightarrow & F \times \mathbf{z}_{2} X / F_{0} \times{ }_{\mathbf{z}_{2}} X,
\end{array}
$$

where the bottom map is induced by the equivariant inclusion $D \tilde{V} \hookrightarrow F$.

(2.10) Proposition. $i_{*}: \tilde{H}_{*}\left(T\left(-\xi_{L}\right)\right) \stackrel{\cong}{\rightarrow} \tilde{H}_{*}\left(E / E_{0}\right)$.

Proof. $i$ induces a map of relative Serre spectral sequences, and at the $E_{2}$-level (homology with local coefficients) $i_{*}: H_{*}\left(B ; H_{*}(D \tilde{V}, S \tilde{V})\right) \rightarrow H_{*}\left(B ; H_{*}\left(F, F_{0}\right)\right)$ is an isomorphism since $i: D \tilde{V} / S \tilde{V} \rightarrow F / F_{0}$ is a homotopy equivalence. The proposition follows.

(2.11) COROllaRY. For a two-fold cover the cofibre $\mathscr{C}$ of the transfer map is homotopy equivalent to $\Sigma^{1-l} T\left(-\xi_{L}\right)$.

(2.12) Corollary. For a two-fold cover that is $h^{*}$-orientable, $h^{*}(\mathscr{C})$ is a free $h^{*}(B)$-module on a generator $u_{0} \in h^{0}(\mathscr{C})$.

REMARK. In the special case of the usual two-fold cover $p: S^{N} \rightarrow R P^{N}$, the cohomology of the cofibre $H^{*}\left(\mathscr{C} ; \mathbf{Z}_{2}\right)$ turns out to be cyclic over the mod 2 Steenrod algebra. In fact, if we write $H^{*}\left(R P^{N} ; \mathbf{Z}_{2}\right)=\mathbf{Z}_{2}[z] / z^{N+1}$, then $\operatorname{Sq}^{i} u_{0}=$ $z^{i} u_{0}$.

\section{BIBLIOGRAPHY}

[A] J. F. Adams, Infinite loop space theory, Ann. of Math. Stud., Princeton Univ. Press, Princeton, N. J., 1978.

[At] M. F. Atiyah, Characters and cohomology of finite groups, Inst. Hautes Études Sci. Publ. Math. 9 (1961), 23-64.

[BG] J. C. Becker and D. H. Gottlieb, The transfer map and fibre bundles, Topology 14 (1975), 1-12.

[D] E. Dyer, Cohomology theories, Benjamin, New York, 1969.

[M] John Milnor, Construction of universal bundles. II, Ann. of Math. (2) 63 (1956), 430-436.

[W] G. W. Whitehead, Elements of homotopy theory, Graduate Texts in Math., Vol. 61, Springer-Verlag, 1978.

Department of Mathematics, California State University, Fresno, California 93710 